\title{
Pregnancy of unknown location: outcome in a tertiary care teaching hospital
}

\author{
A. Shanti Sri*, P. Kalpana \\ Department of Obstetrics and Gynecology, Princess Esra Hospital Deccan College of Medical Sciences Hyderabad,
} Telangan, India

Received: 15 May 2017

Accepted: 20 May 2017

*Correspondence:

Dr. A. Shanti Sri,

E-mail: shantisrinivas@gmail.com

Copyright: ( $\odot$ the author(s), publisher and licensee Medip Academy. This is an open-access article distributed under the terms of the Creative Commons Attribution Non-Commercial License, which permits unrestricted non-commercial use, distribution, and reproduction in any medium, provided the original work is properly cited.

\section{ABSTRACT}

Background: A pregnancy of unknown location (PUL) is a descriptive term used to classify a woman when she has a positive pregnancy test but no intra- or extra-uterine pregnancy is visualized on transvaginal sonography. The objective of present study was to find out the outcome of women with pregnancy of unknown location presenting to a tertiary care teaching hospital.

Methods: The prospective study was conducted from from $1^{\text {st }}$ October 2015 to $31^{\text {st }}$ September 2016, to antenatal outpatient department, at Princess Esra Hospital, Deccan College of medical sciences, Hyderabad. Data was collected for women with early pregnancy or with history of amenorrhea, bleeding or pain. These women were investigated with serum beta-human chorionic gonadotrophin levels at interval of $48 \mathrm{hrs}$ and transvaginal ultrasonography. Expectant management was done for failing pregnancy of unknown location while medical or surgical management was considered for persistent pregnancy of unknown location and ectopic pregnancy.

Results: During study period, 9210 patients were admitted, and, of them, $960(10.42 \%)$ were patients with early pregnancy. Meeting the inclusion criteria were $112(11.6 \%)$ patients who formed the study sample. There were 104 (92.85\%) patients presenting with amenorrhea, $98(87.5 \%)$ had bleeding and $78(69.64 \%)$ presented with pain. Outcome of $42(48.83 \%)$ patients was failing pregnancy, $31(36.04 \%)$ had intrauterine pregnancy, $8(9.3 \%)$ converted to ectopic pregnancy, while $5(5.81 \%)$ had persistent pregnancy of unknown location. All patients with persistent pregnancy of unknown location and 3 patients with ectopic pregnancy were medically treated. Three patients having an ectopic pregnancy were managed surgically.

Conclusions: Management of choice for asymptomatic patients having pregnancy of unknown location is expectant management. Most of the patients suspected to have PUL resolved either into F-PUL or IUP with expectant management.

Keywords: Ectopic pregnancy, Failing pregnancy, Pregnancy of unknown location

\section{INTRODUCTION}

A pregnancy of unknown location (PUL) is a descriptive term used to classify a woman when she has a positive pregnancy test but no intra- or extra-uterine pregnancy is visualized on transvaginal sonography. Pregnancy of unknown location (PUL) is a descriptive term used for a woman who has positive pregnancy test, but no pregnancy can be visualized on transvaginal ultrasonography (TVS). ${ }^{1}$ Incidence of PUL is $8-10 \%$ but some studies have reported incidence of $8-31 \% .^{2}$ PUL has four possible outcomes; a) Intrauterine pregnancy (IUP), b) Failing PUL (F-PUL), c) Ectopic pregnancy (EP), and d) Persistent PUL (P-PUL). ${ }^{3}$ Most common outcome is an F-PUL (44-69\%) and 7-20\% will subsequently be diagnosed with EP . A balance between late diagnosis of EP and over-treatment of possible IUP has to be made. Late diagnosis of EP can lead to increased morbidity, 
mortality and can have effect on future fertility of the woman. PULs form a significant amount of the workload and risk associated with patients attending a hospital with complications in early pregnancy. PUL outcome can be predicted by measuring serum beta-human chorionic gonadotrophin $(\beta$-hCG) hormone and ultrasonography (USG). ${ }^{4}$ Single value of serum hCG to predict outcome in a PUL is of limited value. ${ }^{5}$ The concept of combined USG with serum $\beta$-hCG using discriminatory zone has been widely evaluated. It refers to defined levels of $\beta$ hCG above which gestational sac should be visible on USG with sensitivity approaching $100 \% .^{3}$ With introduction of high resolution TVS, the discriminatory levels of serum $\beta$-hCG $1000-2400 \mathrm{iu} / \mathrm{L}$ has been used. ${ }^{2}$ It is assumed that if serum $\beta$-hCG is above a certain discriminatory level and no IUP visualized on scan, the woman may have EP. TVS must be performed meticulously as pitfalls in image interpretation can lead to false results. $^{2}$ According to a meta-analysis, serum progesterone level is good in predicting the viability of pregnancy but its discriminative capacity is insufficient to diagnose ectopic pregnancy with certainty. ${ }^{5}$ Hence, NICE guidelines 2012 recommend not to use serum progesterone measurements as an adjunct to diagnose either viable intrauterine pregnancy or ectopic pregnancy. ${ }^{6}$

\section{METHODS}

This is a retrospective study over a period of one year reviewing on cases diagnosed as PUL in antenatal outpatient department, from $1^{\text {st }}$ October 2015 to $31^{\text {st }}$ September 2016 at Princess Esra Hospital, Deccan College of medical sciences, Hyderabad. All patients presented with early pregnancy or with history of amenorrhoea, pain or bleeding were considered for inclusion. These patients were investigated by serum $\beta$ $\mathrm{hCG}$ and TVS. The discriminatory zone for the $\beta$-hCG was taken at $1500 \mathrm{IU} / \mathrm{L}$. TVS helped to rule out IUP, free fluid in cul-de-sac and adnexal mass, including EP. Patients who were haemodynamically unstable were excluded. A proforma was filled in which relevant patient information were recorded keeping in view different risk factors for EP. All patients in the study were followed by clinical assessment, serial $\beta$-hCG and TVS to monitor the outcome. Patients were given information sheet about PUL, its outcome, treatment options and need of followup. The management was done according to clinical assessment, compliance and the wishes of the patients. Expectant management was done in PUL patients with decreasing $\beta$-hCG values. These patients were followed every 48hours for $\beta$-hCG and weekly for TVS. Medical treatment was given to the patients with three $\beta$-hCG values at plateau, haemodynamically stable and compliant to treatment. Medical treatment was done with injection Methotrexate $1 \mathrm{mg} / \mathrm{kg}$ given intra-muscularly (IM) after excluding contraindications. Surgical management was considered in patients who were haemodynamically unstable, not compliant or failed to resolve with medical treatment or refused injection methotrexate. These patients were admitted and they underwent laparoscopy or laparotomy, and salpingostomy or salpingectomy was done according to the clinical assessment.

\section{RESULTS}

During the study period, 9210 patients were admitted, and, of them, $960(10.42 \%)$ were patients with early pregnancy. Patients seen with or without symptoms and labelled as PUL were 112 (11.6\%).

Table 1: Outcome of pregnancy of unknown location $(n=86)$.

\begin{tabular}{|ll|l|}
\hline Outcome & Number & Percent \\
\hline Ectopic & 8 & 9.3 \\
\hline Failing PUL & 42 & 48.83 \\
\hline Intrauterine pregnancy & 31 & 36.04 \\
\hline Persistent PUL & 5 & 5.81 \\
\hline
\end{tabular}

Out of these patients, $26(23.2 \%)$ were lost to follow-up or their files were missing at the time of data analysis. The final sample comprised $86(76.78 \%)$ valid patients. These patients were divided into four groups according to their outcome (Table 1).

Table 2: Age group.

\begin{tabular}{|lll|}
\hline Age group & Number & Percent \\
\hline $18-25$ & 29 & 33.72 \\
\hline $26-35$ & 44 & 51.16 \\
\hline $36-45$ & 13 & 15.11 \\
\hline
\end{tabular}

Overall, $73(84.88 \%)$ were young patients (range: 17-35 years). Gestational age of $73(84.88 \%)$ patients was 4-6 weeks. Only $13(15.11 \%)$ patients had gestational age of 7 weeks. A total of $80(93.02 \%)$ patients presented with amenorrhea, while bleeding was found in $77(89.53 \%)$ and pain abdomen in $67(77.9 \%)$ patients. Previous history of ectopic was found in $6(6.97 \%)$ patients.

Table 3: Gestational age.

\begin{tabular}{|lll|}
\hline Gestational age & Number & Percent \\
\hline $4-5$ & 19 & 22.09 \\
\hline $5-6$ & 30 & 34.88 \\
\hline $6-7$ & 24 & 27.9 \\
\hline $7-8$ & 13 & 15.11 \\
\hline
\end{tabular}

Out of these, $42(48.83 \%)$ patients belonged to sub-group having F-PUL. There were $8(9.3 \%)$ who were diagnosed to have EP with appearance of adnexal mass. Among these, $\beta$-Hcg level of two patients decreased and expectant management resolved EP. Three patients underwent medical treatment with Methotrexate after ruling out contraindications, which resolved with a single dose of Methotrexate single dose, two patients underwent surgical management. Five $(5.81 \%)$ patients whose 
outcome was P-PUL were managed by medical treatment and all (100\%) were resolved with single dose of Methotrexate.

Table 4: Symptoms and history.

\begin{tabular}{|lll|}
\hline Symptom & Number & Percent \\
\hline Pain abdomen & 67 & 77.9 \\
\hline Bleeding per vaginum & 77 & 89.53 \\
\hline Amenorrhea & 80 & 93.02 \\
\hline Previous history of ectopic & 6 & 6 \\
\hline
\end{tabular}

\section{DISCUSSION}

It is important in evaluation of a woman with symptomatic first-trimester pregnancy to obtain history about her symptoms, and quantitative hCG value. TVS of uterus and adnexa is also needed if indicated. Some of the women who are diagnosed as having PUL at their first visit can be observed as out-patients with serial $\beta$-hCG and TVS until definitive diagnosis can be made. Expectant management with follow-up of 48 hours can be done for women having PUL with minimal or no symptoms as they are at risk of having ectopic pregnancy. Improved clinical prediction of final outcome of PUL can potentially decrease the number of visits to outpatient as well as shorten the time for some patients to reach a definitive diagnosis. It is well known that patients at risk of having EP require timely and accurate diagnosis as delay in diagnosis of EP can lead to increased morbidity and mortality. However, early intervention may be unnecessary and could harm early IUP. A balance between frequent testing and risk of EP with its complications should be made as frequent testing can lead to falser positive results. ${ }^{7}$ Depending upon the risk factors and clinical examination, individual assessment of the patient may reduce morbidity and mortality. Our study observed the outcome of the patients who presented with amenorrhea, bleeding per vaginum and pain abdomen in first trimester and initially labeled as having PUL. Some of these patients were managed as outpatients according to their symptoms, previous history of EP, $\beta$-hCG level and compliance for follow-up. These patients were called every 48 hours for $\beta$-hCG and weekly for TVS to observe the outcome of PUL. The patients who needed admission because of their symptoms, prior history and $\beta$-hCG value, were managed accordingly. A woman with EP who presents with PUL is likely to have a lower risk of rupture than a woman in whom EP is visualized on TVS initially.

The most common outcome of PUL (44-69\%) is F-PUL. ${ }^{8-}$ ${ }^{13}$ In present study comparable results of $48.83 \%$ were observed for F-PUL. Early IUP may not be visible on TVS due to small size leading to diagnosis of PUL. ${ }^{3,14}$ Various studies have found $30-37 \%$ patients having IUP after initial diagnosis of PUL. ${ }^{3}$ In present study comparable result of $31.7 \%$ was observed for IUP after initial diagnosis of PUL. Various studies have found 8.1$42.8 \%$ of PUL patients having outcome as EP. Lower values (8-14\%) have been observed in specialised screening units when diagnosis of EP was based on the visualisation of adnexal mass rather than the absence of intrauterine sac on TVS. $^{5}$ In present study $9.3 \%$ were diagnosed to be ectopic after an initial diagnosis of PUL. P-PUL accounts for approximately $2 \%$ of total PUL population. In present study, this was found to be higher at about $5 \%$. There is limited data available regarding medical treatment of persistent PUL. In this study, we did not choose other methods of diagnosis, like serum progesterone, tumour markers and mathematical models. These methods have been shown to increase the diagnosis and predict the outcome of PUL as in the study by Thida et al. ${ }^{15}$

\section{CONCLUSION}

Asymptomatic PUL should be managed conservatively as none of the methods to predict the clinical outcome of PUL is $100 \%$ accurate. It is advisable to follow up with hCG and transvaginal ultrasound assessments until the pregnancy is located accurately or intervention becomes necessary. The majority of outcome of PUL is not an ectopic pregnancy. In present study, the rate of ectopic pregnancy in PUL patients is only 9.3\% which is comparable to literature. Expectant management is shown to be safe and also reduces the need for unnecessary surgical intervention with no serious adverse outcomes and to avoid the unnecessary termination of a viable pregnancy. It is associated with increased workload for further blood test for beta hCG and repeat pelvic scans and multiple follow ups that increases the patient anxiety

Generally, as long as patient is haemodynamically stable, further management of PUL could be expectant management. Surgical intervention should be considered only in those with haemodynamically unstable condition and high suspicion for ectopic pregnancy.

Further management for outcome of PUL is based upon good history, clinical examination, haemodynamic stability and patient's informed choices and should not be solely on the basis of doubling time of beta hCG and an empty uterus.

Funding: No funding sources

Conflict of interest: None declared

Ethical approval: The study was approved by the Institutional Ethics Committee

\section{REFERENCES}

1. Kirk E, Condous G, Bourne T. Pregnancies of unknown location. Best Pract Res Clin Obst Gynaecol. 2009;23:493-9.

2. Royal College of Obstetricians and Gynaecologists. The management of early pregnancy loss. Green-top guidelineno. London. RCOG. 2006:1-18. 
3. Sagili H, Mohamed K. Pregnancy of unknown location: an evidence-based approach to management. Obstet Gynaecol. 2008;10:224-30.

4. Romero R, Kadar N, Jeanty P, Copel JA, Chervenak FA, DeCherney A et al. Diagnosis of ectopic pregnancy: Value of the discriminatory human chorionic gonadotropin zone. Obstet Gynecol. 1985;66:357-60.

5. Condous G, Kirk E, Lu C, Van Huffel S, Gevaert O, De Moor B et al. Diagnostic accuracy of varying discriminatory zones for the prediction of ectopic pregnancy in women with a pregnancy of unknownlocation. Ultrasound Obstet Gynecol. 2005;26:770-5.

6. Ectopic pregnancy and miscarriage: diagnosis and initial management. (2012) NICE guidelines [CG154].

7. Bottomley C, VanBelle V, Mukri F, Kirk E, VanHuffel S, Timmerman D et al. The optimal timing of an ultrasound scan to assess the location and viability of an early pregnancy. Hum Reprod 2009; 24:1811-7.

8. Hahlin M, Thorburn J, Bryman I. The expectant management of early pregnancies of uncertain site. HumReprod. 1995;10:1223-7.

9. Banerjee S, Aslam N, Zosmer N, Woelfer B, Jurkovic D. The expectant management of women with early pregnancy of unknown location. Ultrasound Obstet Gynecol. 1999;14:231-6.

10. Banerjee S, Aslam N, Woelfer B, Lawrence A, Elson J, Jurkovic D. Expectant management of early pregnancies of unknown: a prospective evaluation of methods to predict spontaneous resolution of pregnancy. BJOG. 2001;108:158-63.

11. Cacciatore B, Ylostalo P, Stenman UH, Widholm O. Suspected ectopic pregnancy: Ultrasound findings and hCG levels assessed by an immunofluorometric assay. Br J Obstet Gynaecol. 1988;95:497-502.

12. Condous G, Lu C, Van Huffel SV, Timmerman D, Bourne T. Human chorionic gonadotrophin and progesterone levels in pregnancies of unknown location. Int J Gynaecol Obstet. 2004;86:351-7.

13. Hajenius PJ, Mol BW, Ankum WM, van der Veen F, Bossuyt PM, Lammes FB. Suspected ectopic pregnancy: Expectant management in patients with negative sonographic findings and low serum hCG concentrations. Early Pregnancy.1995;1:258-62

14. Condous G, Okaro E, Khalid A, LuC, VanHuffel S, Timmerman D, et al. A prospective evaluation of a single-visit strategy to manage pregnancies of unknown location. Hum Reprod. 2005;20:1398403

15. Thida W, Shah AA. Review on outcome of pregnancy of unknown location and further management: a retrospective study. J Gynecol Neonatal Biol. 2017;3(1):1-4.

Cite this article as: Sri AS, Kalpana P. Pregnancy of unknown location: outcome in a tertiary care teaching hospital. Int J Reprod Contracept Obstet Gynecol 2017;6:2787-90. 